\title{
Dehydration-induced tps gene transcripts from an anhydrobiotic nematode contain novel spliced leaders and encode atypical GT-20 family proteins
}

\author{
K. Goyal ${ }^{\text {a }}$, J.A. Browne ${ }^{\text {b,c }}$, A.M. Burnell ${ }^{\text {b }}$, A. Tunnacliffe ${ }^{\mathrm{a}, *}$ \\ a Institute of Biotechnology, University of Cambridge, Tennis Court Road, Cambridge CB2 1QT, UK \\ ${ }^{\mathrm{b}}$ Department of Biology, Institute of Bioengineering and Agroecology, National University of Ireland Maynooth, Maynooth, Co Kildare, Ireland \\ ${ }^{\mathrm{c}}$ Department of Clinical Medicine, Nutrigenomics Research Group, Institute of Molecular Medicine, Trinity Centre for Health Sciences, \\ St. James Hospital, Dublin 8, Ireland
}

Received 7 October 2004; accepted 21 January 2005

Available online 03 March 2005

\begin{abstract}
Accumulation of the non-reducing disaccharide trehalose is associated with desiccation tolerance during anhydrobiosis in a number of invertebrates, but there is little information on trehalose biosynthetic genes in these organisms. We have identified two trehalose-6-phosphate synthase (tps) genes in the anhydrobiotic nematode Aphelenchus avenae and determined full length cDNA sequences for both; for comparison, full length tps cDNAs from the model nematode, Caenorhabditis elegans, have also been obtained. The A. avenae genes encode very similar proteins containing the catalytic domain characteristic of the GT-20 family of glycosyltransferases and are most similar to tps-2 of C. elegans; no evidence was found for a gene in A. avenae corresponding to Ce-tps-1. Analysis of A. avenae tps cDNAs revealed several features of interest, including alternative trans-splicing of spliced leader sequences in Aav-tps-1, and four different, novel SL1-related transspliced leaders, which were different to the canonical SL1 sequence found in all other nematodes studied. The latter observation suggests that A. avenae does not comply with the strict evolutionary conservation of SL1 sequences observed in other species. Unusual features were also noted in predicted nematode TPS proteins, which distinguish them from homologues in other higher eukaryotes (plants and insects) and in micro-organisms. Phylogenetic analysis confirmed their membership of the GT-20 glycosyltransferase family, but indicated an accelerated rate of molecular evolution. Furthermore, nematode TPS proteins possess N- and C-terminal domains, which are unrelated to those of other eukaryotes: nematode C-terminal domains, for example, do not contain trehalose-6-phosphate phosphatase-like sequences, as seen in plant and insect homologues. During onset of anhydrobiosis, both tps genes in A. avenae are upregulated, but exposure to cold or increased osmolarity also results in gene induction, although to a lesser extent. Trehalose seems likely therefore to play a role in a number of stress responses in nematodes.

(c) 2005 Elsevier SAS. All rights reserved.
\end{abstract}

Keywords: Anhydrobiosis; Desiccation tolerance; Nematode; Trehalose synthase; Spliced leader; Accelerated evolution

\section{Introduction}

Water is considered a prerequisite for life, but some organisms survive desiccation for indefinite periods by entering a state of suspended animation. This ability, called anhydrobiosis ("life without water"), occurs across all biological king-

\footnotetext{
* Corresponding author. Institute of Biotechnology, University of Cambridge, Tennis Court Road, Cambridge CB2 1QT, UK. Tel.:

+44 1223 766549; fax: +44 1223334162 .

E-mail address: at10004@biotech.cam.ac.uk (A. Tunnacliffe).
}

doms, including bacteria, fungi, animals, and plants. Members of three invertebrate taxa, bdelloid rotifers, tardigrades and nematodes, can undergo anhydrobiosis at all stages of the life cycle; other invertebrates have stage-specific anhydrobiotic forms (reviewed in Ref. [1]). The molecular mechanisms governing anhydrobiosis are not well understood, but there is considerable interest in the role of the non-reducing disaccharides trehalose (in animals and fungi) and sucrose (in plants). For instance, when Aphelenchus avenae, a soildwelling fungivorous nematode amenable to laboratory culture, is dried slowly to induce anhydrobiosis, it converts up 
to $20 \%$ of its dry weight to trehalose and this correlates with acquisition of desiccation tolerance [2,3], albeit somewhat loosely [4]. Surprisingly, however, bdelloid rotifers seem unable to produce trehalose at all [5], although their anhydrobiotic capability is well established [1]. Nevertheless, the importance of non-reducing disaccharides has been stressed repeatedly, since it is well known that such sugars are able to stabilise dried proteins and membrane systems in vitro and it is widely assumed they have similar stabilising properties in vivo [6].

Three main biosynthetic routes for trehalose are recognised [7], the most common of which involves transfer of glucose from UDP-glucose to glucose-6-phosphate to yield trehalose-6-phosphate, followed by removal of the phosphate to give trehalose; these steps are catalysed by trehalose6-phosphate synthase (EC 2.4.1.15) and trehalose-6phosphate phosphatase (EC 3.1.3.12), respectively. Genes encoding the first of these enzymes ( $t p s$ ) have been identified in a wide range of organisms, including plants (e.g., Selaginella lepidophylla [8]), invertebrates (e.g., Caenorhabditis elegans [9]), yeast (e.g., Saccharomyces cerevisiae [10,11]) and bacteria (e.g., Escherichia coli [12]); trehalose-6phosphate phosphatase genes have also been characterised for many micro-organisms. Although some of these organisms are anhydrobiotic (e.g., Se. lepidophyllum, Sa. cerevisiae), the majority are not; accordingly, in the latter species, the role of trehalose is probably not related to desiccation tolerance. Rather, trehalose might be a general stress molecule, at least in micro-organisms, being linked to multiple stress responses, including those to heat [13,14], cold [15], reactive oxygen species [16] and elevated osmolarity [17].

Thus, although there is significant information on the genetics of trehalose synthesis in desiccation-sensitive organisms, little is known in anhydrobiotic animals, despite its potential importance for them. To begin to redress this imbalance, we have characterised full length cDNAs for tps genes from the nematode $A$. avenae, compared them to counterparts in C.elegans, and examined the regulation of these genes under several stress conditions, including dehydration. We also present data on splicing of tps mRNAs_-including the use of novel spliced leader sequences, and evidence for alternative trans- and cis-splicing - and on the evolution and structure of nematode TPS proteins.

\section{Material and methods}

\subsection{Isolation of tps cDNAs from A. avenae and C. elegans}

A. avenae and C.elegans were grown as described [3,18]. Degenerate PCR primers [5] were used to amplify tps fragments from genomic DNA, isolated as described [3], of both nematode species. From these sequences, specific primers were designed to generate corresponding partial cDNA sequences by reverse-transcriptase PCR (RT-PCR). Total RNA was digested with RQ1 RNase-free DNase (Promega) and purified by phenol/chloroform extraction. RT-PCR was performed in one step with gene-specific primers using the Access RT-PCR system (Promega): $3 \mu$ RNA was used as template according to manufacturer's instructions except that Red Hot DNA polymerase (ABgene) was used. Incubations comprised one cycle of $48{ }^{\circ} \mathrm{C}$ for $45 \mathrm{~min}, 94^{\circ} \mathrm{C}$ for $2 \mathrm{~min}$ to synthesize first strand $\mathrm{cDNA}$ and to denature template, followed by 40 cycles at $94{ }^{\circ} \mathrm{C}$ for $30 \mathrm{~s}, 55{ }^{\circ} \mathrm{C}$ for $1 \mathrm{~min}$ and $68{ }^{\circ} \mathrm{C}$ for $2 \mathrm{~min}$, followed by a final extension at $68{ }^{\circ} \mathrm{C}$ for 7 min to synthesize second strand and amplify DNA. The $5^{\prime}$ and $3^{\prime}$ ends of A. avenae and C. elegans transcripts were identified using the GeneRacer kit (Invitrogen). PCR products were cloned into pCR4-TOPO vector, and transformed into One-Shot chemically competent bacteria (Invitrogen). Full length cDNA was generated, after reverse transcription of total RNA, using gene-specific primers with KOD XL DNA polymerase (Novagen), as follows: $94{ }^{\circ} \mathrm{C}$ for $1 \mathrm{~min}$, followed by 30 cycles at $94^{\circ} \mathrm{C}$ for $30 \mathrm{~s}, 58^{\circ} \mathrm{C}$ for $2 \mathrm{~s}$ and $70{ }^{\circ} \mathrm{C}$ for $4 \mathrm{~min}$, followed by a final extension at $74{ }^{\circ} \mathrm{C}$ for $10 \mathrm{~min}$. Internal primers were designed to sequence all cDNAs on both strands. Southern hybridisations were carried out as described [3], except that probes were detected using an AlkPhos direct labelling and detection system (Amersham Pharmacia Biotech). Fragments corresponding to nucleotides 1280-1990 of Aav-tps-1 complete cDNA sequence and nucleotides 15802290 of $C e$-tps- 1 complete cDNA sequence were used as probes.

\subsection{Phylogenetic analysis}

Apart from the A. avenae and C. elegans TPS sequences derived for this paper, sequences were retrieved from GenBank (http://www.ncbi.nlm.nih.gov) or the CAZy database of GT-20 family members (http://afmb.cnrs-mrs.fr/CAZY). Their species and accession numbers are A. thaliana TPS1 (CAA69879), Se. lepidophylla (U96736), Sa. cerevisiae (CAA85083), Apis mellifera (XP_392397), D. melanogaster (NP_608827), E. coli (BAA15717), A. avenae TPS1 (AJ811568), A. avenae TPS2 (AJ811570), C. elegans TPS2 (AJ811574), C. elegans TPS1 (AJ811573), C. briggsae TPS1 (CAE58687), Candida glabrata (XM_448136), Sc. pombe (Z29971.1), Neurospora crassa (XM_330364) and Sinorhizobium meliloti (AE007206.1).

Multiple alignments were performed with ClustalX [19] using the Gonnet 250 matrix. For pairwise alignments the gap opening penalty was set at 35.0 and the gap extension penalty at 0.75 . For multiple alignments the gap opening penalty was set at 15.0 and the gap extension penalty at 0.30 . The alignment of GT-20 regions used for tree building corresponds to residues 286-710 of Aav-TPS-1. Phylogenetic analyses were carried out on the amino acid alignment using maximum parsimony (MP), maximum likelihood (ML), neighbor joining (NJ) and Bayesian inference. In all cases, E. coli and S. meliloti were used as a monophyletic outgroup. Unweighted MP analyses were carried out with PAUP* $4.0 \mathrm{~b} 10$ [20] using heuristic methods. Gaps in the alignment 
were treated as missing data; the $\mathrm{NJ}$ tree was constructed using the same program. The ML phylogenetic tree was constructed using the WAG model of amino acid substitution [21] as implemented in Tree-Puzzle 5.2 (www.tree-puzzle.de; [22]). The data set was tested against all available models of protein evolution in Tree-Puzzle 5.2; the WAG model gave the best ML score. Bayesian trees were constructed using MrBayes 3.0B4 [23]. A 5,000,000 generation Monte Carlo Markov chain was carried out with sampling every 100th generation. The first 500 trees were discarded and the remaining trees were combined into a single file which was imported into PAUP* $4.0 \mathrm{~b} 10$ to compute the $50 \%$ majority rule consensus tree. Nodal support for the MP and NJ trees was estimated using bootstrap analyses based on 1000 replicates with PAUP* programs. Bootstrap supports for the ML tree were obtained using puzzleboot (www.tree-puzzle.de/ puzzleboot.sh), which determines the ML distance matrices of appropriately resampled data partitions. Posterior probabilities for the Bayesian consensus tree were determined using the sumt command of MrBayes 3.0B4.

\subsection{Quantitative real-time reverse transcriptase PCR}

Quantitative PCR and stress treatment of nematodes were performed as described [3]. To generate gene-specific realtime PCR standards, primers were designed that amplified a small region ( $125 \mathrm{bp})$ of the target (Aav-tps-1 and Aavtps-2) and control (Aav-ama-1) genes. Specific forward primers were used for Aav-tps-1 (5'-GAG CAG CAT TTG CAT ACA AAA AC-3') and Aav-tps-2 (5'-GAG TTT ACG TAC GAA CAA ATT GG-3') together with a common reverse primer (5'-GTT GTG CTG ACC TTA TTC GTC T-3'). Preliminary RT-PCR experiments were performed to establish conditions for gene-specific amplication.

\section{Results}

\subsection{Trehalose-6-phosphate synthase cDNAs from A. avenae and C. elegans}

Full length cDNA clones were obtained for both tps genes, Ce-tps- 1 (chromosome X) and Ce-tps-2 (chromosome II), of C. elegans (accession nos. AJ811573 and AJ811574, respectively). For Ce-tps-1, the cDNA sequence predicts a protein 1331 amino acids in length, with $\mathrm{M}_{\mathrm{r}} 151,000$; for Ce-tps-2, a protein of 1229 amino acids and $\mathrm{M}_{\mathrm{r}}$ 140,000 is predicted. Similar sequences from overlapping cDNA clones were also reported for both genes by Pellerone et al. [9] (accession nos. AJ512332 and AJ512333, respectively).

Two different tps genes were also identified in A. avenae and full length cDNA sequences were determined for both from multiple RT-PCR and RACE clones; $70 \%$ of the cDNA sequence was also checked against genomic sequence, showing that there are at least 17 exons for Aav-tps-1, and at least 15 exons for Aav-tps-2. For Aav-tps-1, alternative trans- splicing of the same spliced leader sequence was observed, giving two mRNAs (AJ811568 and AJ811569, respectively) which differ in the extent of their $5^{\prime}$ ends, with total length 3945 or 3396 bases (excluding spliced leader and polyA tail, but including 3' untranslated region; Fig. 1A), respectively. This alternative splicing gives rise to two forms of protein, 1155 and 980 residues in length, and $M_{r} 131,000$ and 112,000, respectively, assuming cis-splicing is identical; the short form derives from a mRNA which lacks the first four exons. Aavtps-2 mRNA, as derived from overlapping RT-PCR and RACE clones, is 4291 bases long (AJ811570) and encodes a protein of 1303 amino acids in length, with $\mathrm{M}_{\mathrm{r}} 148$ 000; a full length Aav-tps-2 cDNA (clone T3; AJ811571), corresponding to this sequence, has also been obtained. The Aav-tps-2 mRNA is therefore somewhat longer than the equivalent mRNAs from Aav-tps-1; despite repeated attempts by 5'-RACE cloning, we were unable to find evidence for a comparable, longer version of Aav-tps-1 mRNA; nor have we found a very short form of Aav-tps-2 comparable to that of internally transspliced Aav-tps- 1 lacking the first four exons.

An alignment of cDNA sequences of Aav-tps- 1 and Aavtps-2 showed up variation in internal cis-splicing of tps exons (not shown). Thus, although both genes have similar exon structure, as determined by partial genomic sequencing, alternative splice donor sites are used in exons 4 and 5. While for Aav-tps-1, exon 4 contributes 185, and exon 5220 bases to the mature mRNA, the equivalent exons of Aav-tps- 2 contribute 233 and 169 bases, respectively; different splice donor positions are used for these exons in the two genes (Fig. 1B). Intriguingly, however, we have also identified a variant, full length cDNA clone (T10; AJ811572) which is most similar to Aav-tps-2 but which displays a splicing pattern like that of Aav-tps-1, suggesting that alternative splicing might occur within the same gene. Thus, variable splicing, both in cis and in trans, appears to take place in A. avenae tps pre-mRNAs.

\subsection{Multiple novel spliced leader sequences in Aav-tps-2 mRNAs}

Unusually, although alternative trans-splicing was not observed in $A a v$-tps-2, four variant spliced leader sequences were found in RACE cDNA clones from this gene, one of which, SL1a, was identical to that used in Aav-tps-1; SL1a was found in three independent cDNA clones from two different genes. SL1a differs from C. elegans SL1 in two positions out of 22; in fact, none of the $A$. avenae SL sequences was identical to those of $C$. elegans and other nematode species, although one variant (SL1b) differs from the canonical SL1 by only a single base (Table 1 ). All four A. avenae spliced leaders are related to SL1 since they have the characteristic block of eight $A$ or $T$ residues after the initial $\mathrm{G}$ dimer, and all end in TTTGAG, like $C$. elegans SL1. In contrast, C. elegans SL2 and all other minor spliced leaders from this nematode are characterised by a block of six A or T residues after the initial GG; their sequences also all end in PyAAG, usually CAAG. The variability in A. avenae spliced leader sequences 
A

\begin{tabular}{|c|c|c|}
\hline s-1 & long & ETTT.T. \\
\hline av-tps-1 & long & TGCAGCACAGCTTCGACACCAACGAGTTCTTCGAGAAGTTCGTCAATGCG \\
\hline av-tps-1 & long & CTCGGCTACCATACGGTGCAGTTCTGGCGCGCCGGAGTGCCGTACGTCTT \\
\hline av-tps-1 & long & $\begin{array}{rllllllllllll}\text { M } & \text { S } & \text { H } & \text { G } & \text { T } & \text { K } & \text { Y } & \text { R } & \text { D } & \text { A } & \text { L } & \text { L } & \text { F } \\
\text { CGACTCGGACATGTCGCACGGGACGAAGTACCGCGACGCGCTCCTCTTCA }\end{array}$ \\
\hline$a v-t p s-1$ & long & $\begin{array}{lllllllllllllllll}S & L & T & L & Y & D & V & N & T & G & K & S & R & L & K & E & L \\
\text { GCCTCACCCTCTACGACGTCAACACCGGCAAGAGCCGGCTCAAGGAGCTC }\end{array}$ \\
\hline av-tps-1 & long & 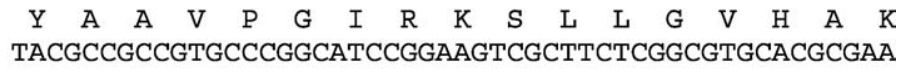 \\
\hline$a v$ & long & $\begin{array}{cccccccccccccccc}R & F & G & E & Q & Y & H & H & L & Q & R & R & R & S & V & S \\
\text { GCGCTTCGGAGAGCAGTACCACCACCTTCAGCGACGTCGCTCCGTCTCGT }\end{array}$ \\
\hline$a v-t p s-1$ & long & 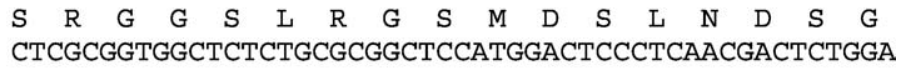 \\
\hline$a v-t$ & long & 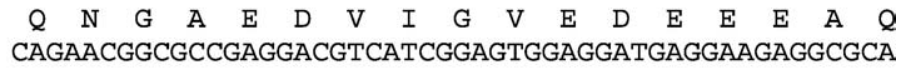 \\
\hline$a v-t p s-1$ & long & $\begin{array}{cccccccccccccccc}\mathrm{K} & \mathrm{F} & \mathrm{R} & \mathrm{G} & \mathrm{K} & \mathrm{R} & \mathrm{T} & \mathrm{S} & \mathrm{I} & \mathrm{S} & \mathrm{L} & \mathrm{D} & \mathrm{P} & \mathrm{A} & \mathrm{A} & \mathrm{A} \\
\text { GAAGTTCCGCGGCAAACGGACCTCGATATCGCTGGATCCCGCGGCGCCG }\end{array}$ \\
\hline & & $\begin{array}{lllllllllllllllll}\text { G } & \text { E } & \text { V } & \text { M } & \text { F } & \text { T } & \text { I } & \text { E } & \text { D } & \text { G } & \text { A } & \text { C } & \text { F } & \text { P } & \text { S } & \text { G } & \text { G } \\
\text { GCGAAGTGATGTTCACCATCGAGGATGGCGCGTGTTTCCCGTCGGCGC }\end{array}$ \\
\hline Aav-tps-1 & short & \\
\hline & & $\begin{array}{ccccccccccccccccc}\text { L } & \text { A } & N & \text { T } & \text { H } & \text { F } & \text { Q } & \text { Q } & \text { R } & \text { V } & \text { I } & \text { N } & \text { V } & \text { S } & \text { N } & \text { A } & \text { P } \\
\text { CTCGCAACACGCACTTCCAAGCAACGCGTGATCAACGTCTCCAACGCGCC }\end{array}$ \\
\hline$-\operatorname{tp}$ & & ACCCAAGTTTGAG $C A A C G C G T G A T C A A C G T C T C C A A C G C G C C$ \\
\hline
\end{tabular}

$\begin{array}{llllllllllllllll}P & V & S & L & K & R & E & K & S & G & E & W & E & I & K & Q\end{array}$

Aav-tps-1 long GCCGGTCTCGCTGAAGCGGGAGAAGTCCGGCGAGTGGGAGATCAAGCAGG Aav-tps-1 short GCCGGTCTCGCTGAAGCGGGAGAAGTCCGGCGAGTGGGAGATCAAGCAGG

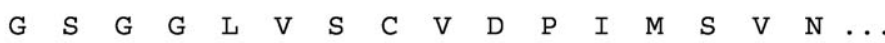
Aav-tps-1 long GCTCCGGCGGGCTCGTCTCGTGCGTCGACCCGATCATGTCCGTCAAC... Aav-tps-1 short GCTCCGGCGGGCTCGTCTCGTGCGTCGACCCGATCATGTCCGTCAAC... M $S \quad \mathrm{~V} \quad \mathrm{~N} \ldots$

B

Aav-tps-1 (RT-PCR), Aav-tps-2 (T10)

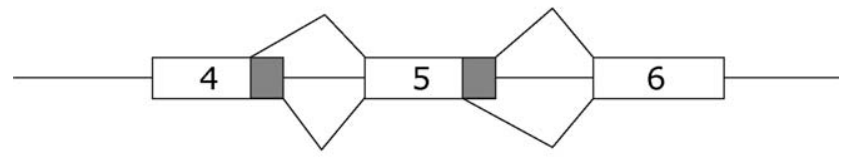

Aav-tps-2 (RT-PCR), Aav-tps-2 (T3)

Fig. 1. Analysis of A. avenae tps mRNA splicing patterns. (A) Alignment of the 5 ' ends of the long and short forms of Aav-tps- $1 \mathrm{cDNA}$ sequences. Splice donor and acceptor sites are indicated with a solid underline, spliced leader sequence by a dotted underline. Methionine residues are indicated by bold lettering. The first initiation codon in the long form of Aav-tps- 1 is not in frame with the major ORF. (B) The use of alternative splice donor sequences in exons 4 and 5 of A. avenae tps genes is shown, with shading representing the different extents of the exon sequences found in cDNAs (not to scale). RT-PCR clones from Aav-tps-1, and the Aav-tps-2 full length cDNA clone T10, contain short exon 4 and long exon 5 sequences, whereas most RT-PCR clones from Aav-tps-2, and the Aav-tps-2 full length cDNA clone T3, contain long exon 4 and short exon 5 sequences.

is striking, since in all other nematode species examined, the SL1 sequence is absolutely conserved [24], with one exception where a SL1 variant differing by a single base was seen $[25,26]$.

\subsection{Diversity of tps genes in A. avenae and C. elegans}

The predicted protein sequences from $A$. avenae tps genes (Aav-TPS-1 and Aav-TPS-2, respectively) are extremely simi- 
Table 1

Variant spliced leader sequences of A. avenae tps mRNAs

\begin{tabular}{|c|c|c|c|c|}
\hline & Species & SL sequence & Length (bp) & mRNA \\
\hline SL1a & A. avenae & GGTTTATATACCCAAGTTTGAG & 22 & $A a v-t p s-1 ; A a v-t p s-2$ \\
\hline SL1b & A. avenae & GGTTTTATTACCCAAGTTTGAG & 22 & Aav-tps-2 \\
\hline SL1c & A. avenae & GGTTTAAATACCCAAATTTGAG & 22 & $A a v-t p s-2$ \\
\hline SL1d & A. avenae & GGTTTAAATACCCTAATTTGAG & 22 & $A a v-t p s-2$ \\
\hline SL1 & C. elegans & GGTTTAATTACCCAAGTTTGAG & 22 & \\
\hline SL2 & C. elegans & GGTTTTAACCCAGTTACTCAAG & 22 & \\
\hline
\end{tabular}

lar: the major differences are at the N-terminus, where AavTPS-2 is longer than the longest form of Aav-TPS-1 by 149 residues, and in the alternatively cis-spliced region noted above. The remaining protein sequences are identical apart from four amino acid replacements. Intriguingly, both A. avenae tps genes are homologues of tps-2 from C. elegans; to date, we have not found a counterpart of $C$. elegans tps-1. At the protein level, Aav-TPS-2 and Ce-TPS-2 show $62 \%$ similarity, whereas only $49 \%$ similarity is seen between Aav-TPS2 and Ce-TPS-1; an alignment of both C. elegans TPS sequences with each other shows only $51 \%$ similarity. Therefore, TPS-2 sequences from both nematodes show approximately the same evolutionary distance from Ce-TPS-1. One interpretation of this observation is that the two tps genes represented in C. elegans diverged before the evolutionary separation of the two species of nematode, after which the gene equivalent to Ce-tps-2 in A. avenae was reduplicated to give Aav-tps- 1 and Aav-tps-2.

Southern hybridisation results are consistent with the lack of a Ce-tps- 1 homologue in A. avenae. Fig. 2A shows genomic DNA from both nematode species digested with EcoRI and hybridised under low stringency conditions with a cDNA sequence representing part of the Ce-tps- 1 coding region. While two hybridising bands are clearly visible in C. elegans DNA, presumably corresponding to Ce-tps-1 and Ce-tps-2, no fragments were detected in the A. avenae DNA digest.
When A. avenae DNA digested with a range of restriction enzymes is hybridised with a probe from one of its own genes, Aav-tps-1, two bands are seen in most tracks, consistent with the presence of two tps genes (Fig. 2B).

\subsection{Comparison and phylogenetic analysis of TPS protein sequences}

TPS sequences comprise glycosyltransferase family 20 (GT-20) of carbohydrate active enzymes (CAZy; afmb.cnrs$\mathrm{mrs}$.fr/CAZY/GT_20.html). The type sequence is OtsA from E. coli, which is folded into two domains and assembled as a tetramer [27]; most prokaryotic TPS sequences in the CAZy database are similar to OtsA in length and sequence, and therefore probably share this structure. The central region of nematode TPS proteins, and equivalent sequences from other eukaryotic TPS proteins, can be aligned with OtsA; we refer to this common sequence as the GT-20 region.

Amino acid alignments of GT-20 regions from 15 microbial, plant and animal TPS sequences, including those of the nematodes described above, were made with ClustalX and phylogenetic analyses were carried out using four methods: maximum parsimony (MP), maximum likelihood (ML), neighbor joining (NJ) and Bayesian inference. Bacterial sequences from $E$. coli and S. meliloti were defined as a monophyletic outgroup. Fig. 3A shows the dendrogram derived

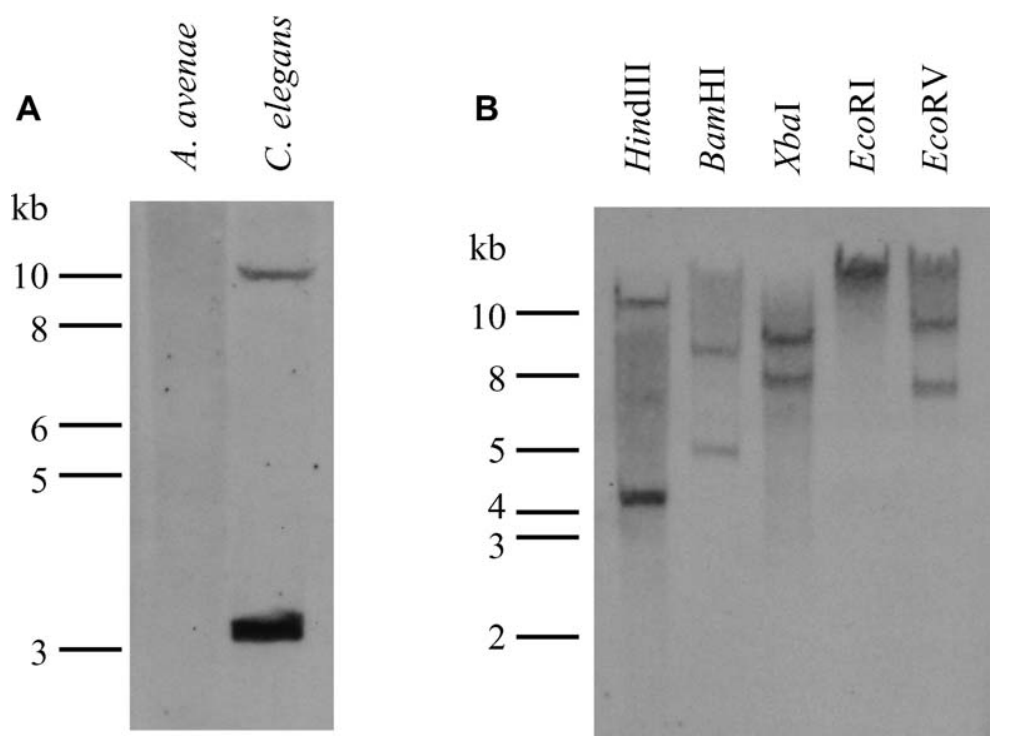

Fig. 2. Lack of a homologue of Ce-tps-1 in the A. avenae genome. (A) Southern hybridisation of EcoRI-digested A. avenae and C. elegans genomic DNAs probed with a $C e$-tps- 1 cDNA fragment. (B) A. avenae genomic DNA digested with HindIII, BamHI, XbaI, EcoRI and EcoRV restriction enzymes, respectively, and probed with a Aav-tps-1 cDNA fragment. 
A

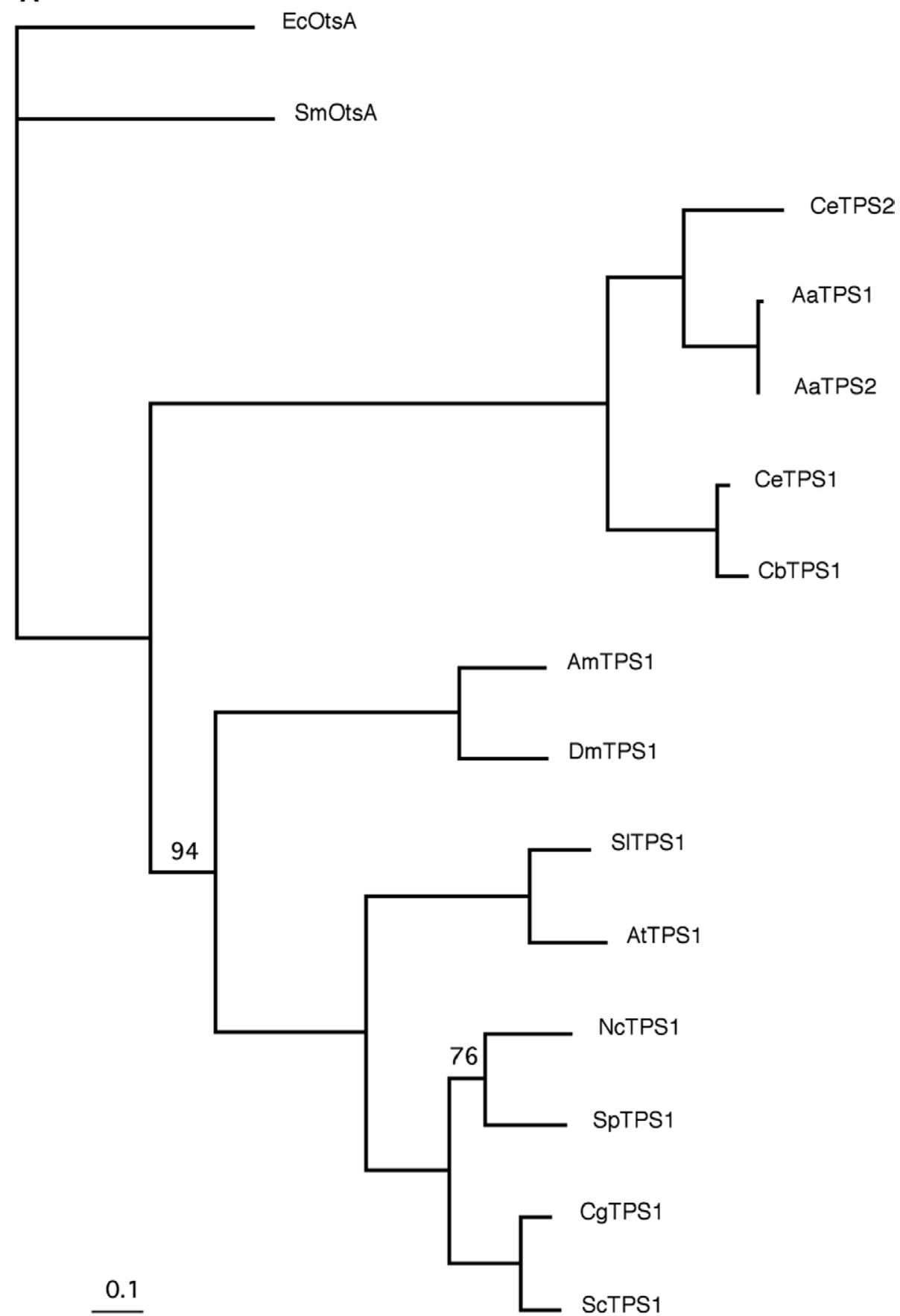

Fig. 3. Evolution and structure of TPS enzymes. (A) Dendrogram constructed from maximum likelihood analysis of a ClustalX alignment of GT-20 region sequences. TPS sequences used are given in Section 2; species names are abbreviated in the figure according to the first letter of genus and species names. The scale bar represents 0.1 substitutions per site. Bootstrap values are 100\% for all nodes, except as indicated. (B) Relative domain length of TPS sequences from various species. Each sequence is divided into N-terminal, GT-20 and C-terminal domains, where relevant, with number of amino acids in each domain shown, of a subset of the species in A. Different shading of the insect and plant C-terminal domains indicates their relatedness to TPP sequences.

from ML analysis, but the same topology was obtained with all phylogenetic reconstruction methods. The tree is robust with bootstrap values of $100 \%$ at most nodes and is in accord with organismal phylogeny. The grouping of $C$. glabrata and $S$. cerevisiae in a single clade is in agreement with recent phylogenetic analyses $[28,29]$. The lower bootstrap support for the grouping of $S$. pombe and $N$. crassa reflects their placement in different clades in the phylum Ascomycota [29].
The phylogenetic analysis confirms the close relationship between nematode TPS sequences (from A. avenae, C. elegans and C. briggsae), which show up to $70 \%$ identity across genera in the GT-20 region, and supports the hypothesis that the two $C$. elegans genes diverged before the evolutionary separation from $A$. avenae, after which a reduplication event occurred in A. avenae to give rise to Aav-tps-1 and Aav-tps-2. Strikingly, as indicated by the ML and NJ phylograms, nema- 
B

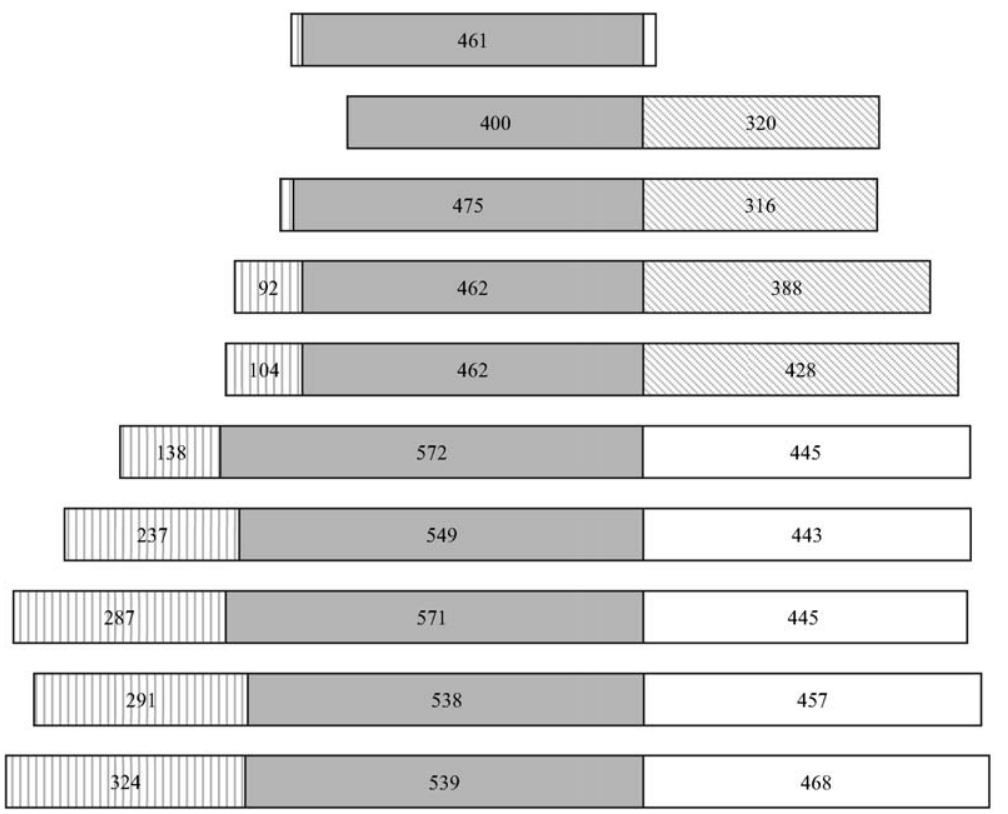

EcOtsA

ScTPS1

AmTPS1

DmTPS1

AtTPS1

SITPS1

AaTPS1

CeTPS2

AaTPS2

CbTPS1

CeTPS1

Fig. 3. (continued)

tode GT-20 region sequences show accelerated evolution in comparison to other homologues, whether from microorganisms, plants or animals. This apparent anomaly of nematode TPS sequences could be due to the accelerated molecular evolution in phylum Nematoda relative to other animal taxa [30]. One unique feature of nematode GT-20 regions which emerges from the alignment is an insertion of approximately 50-80 residues (positions 194-274 of Aav-TPS-1), corresponding largely to exon 5 in Aav-tps-1. Despite these features, the phylogenetic analysis supports the inclusion of the nematode proteins in the GT-20 family of glycosyltranserases and emphasises the sequence conservation of the catalytic core.

As well as the GT-20 region, plant and animal TPS proteins have a large $\mathrm{C}$-terminal domain; nematode and plant TPS proteins also have a substantial $\mathrm{N}$-terminal extension (Fig. 3B). In plants, the $\mathrm{C}$-terminal domain has been reported to show similarity to bacterial trehalose-6-phosphate phosphatase (TPP; exemplified by OtsB from E. coli) suggesting that both activities required for trehalose synthesis are contained within the same protein. Zentella et al. [8] and Vogel et al. [31] were unable to confirm TPP activity encoded by tps genes from S. lepidophylla and A. thaliana, respectively, however. This is perhaps not surprising, since the similarity between the plant C-terminal TPS and OtsB sequences is slight; more extensive is the relatedness between insect C-terminal TPS sequences and OtsB (data not shown), and it is likely that these TPP-related sequences are functional since the Drosophila TPS1 protein alone is able to direct trehalose synthesis in human cells [32]. No relatedness to TPP is apparent in TPS sequences from nematodes, however: BLAST searches with Aav-TPS-2 C-terminal sequences (residues 886-1303) show strong matches with the equivalent regions of $C$. elegans TPS proteins, but no apparent similarity with TPP sequences from micro-organisms or plants, nor with plant or insect TPS proteins (data not shown). Weaker but significant BLAST matches were seen with GOB-1 (Q9XTQ5), a chaperone-like protein in $C$. elegans with possible involvement in growth and locomotion; with a hypothetical protein (NP_662202) from Chlorobium tepidum, a photosynthetic, anaerobic, green-sulphur bacterium; and with hydrolases of the HAD superfamily; the relevance of these matches is unclear at present. The function of the N-terminal domain of nematode TPS proteins is even more of a puzzle, being of variable length and apparently unrelated to other proteins: the N-terminal 250 residues of Aav-TPS-2 show no significant BLAST matches other than with equivalent sequences from other nematodes. In summary, nematode TPS sequences possess several features which distinguish them from those of micro-organisms and other higher eukaryotes and we can categorise TPS proteins into at least three groups accordingly.

\subsection{Regulation of A. avenae tps genes by water stress}

One mechanism by which the trehalose accumulation associated with anhydrobiosis in A. avenae might be achieved is by upregulation of its trehalose synthase genes. To test this, the expression level of both Aav-tps-1 and Aav-tps-2 during dehydration was estimated by quantitative PCR. The specificity of any response was determined by comparison with expression levels under other stress conditions, namely cold and heat shock, and oxidative and osmotic stresses. An absolute quantification method using a gene-specific external standard curve was used to determine the copy number of each gene per $\mu \mathrm{g}$ of total RNA. Primer combinations specific for 


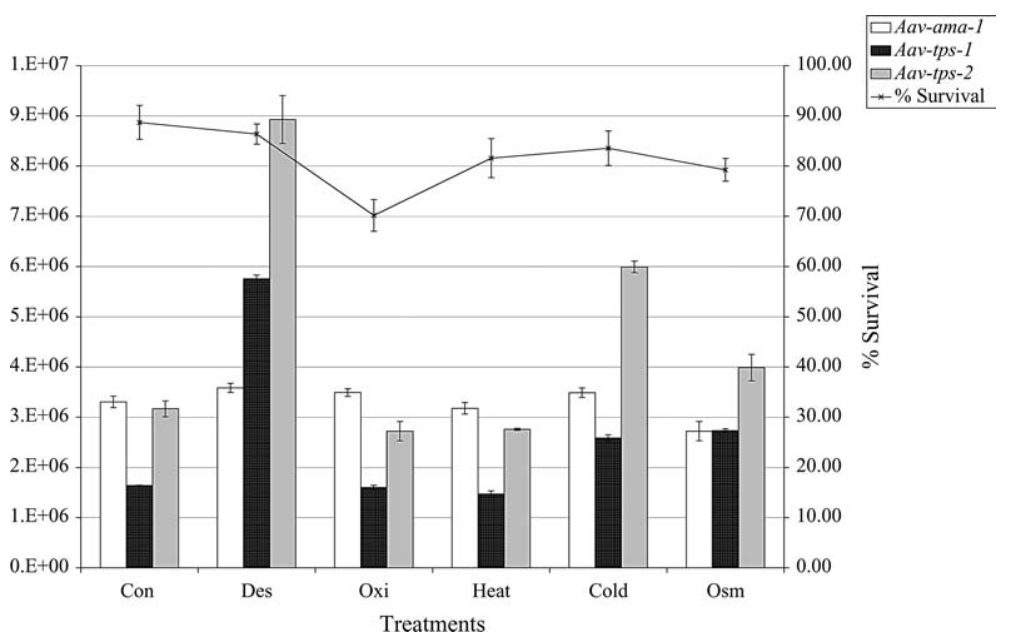

Fig. 4. Stress regulation of A. avenae tps genes. Quantitative PCR analysis of Aav-tps-1, Aav-tps-2 and Aav-ama-1 mRNA from A. avenae under various conditions: desiccation (Des), oxidative (Oxi), heat, cold and osmotic (Osm) stresses were imposed; Con: control, unstressed animals. Survival of nematode populations subjected to the same stresses is also shown.

each $t p s$ gene were chosen, allowing expression of each gene to be assessed separately, as well as for Aav-ama-1, which encodes a subunit of RNA polymerase II, as an internal reference. Fig. 4 shows that Aav-tps- 1 and Aav-tps- 2 mRNA levels are both increased approximately threefold in drying nematodes, and therefore that expression is responsive to water loss. Cold stress and hyperosmotic stress also result in a slight increase in tps gene expression, although less than twofold in each case; in contrast, oxidative and heat stress do not appear to affect expression significantly. Survival of $A$. avenae was comparable for each of the various stresses imposed. Thus, expression of both tps genes is consistent with a role for trehalose in combatting water, and perhaps other, stresses.

\section{Discussion}

Trehalose has been implicated in anhydrobiosis for over 30 years due to its presence in a number of organisms, mainly animals or fungi, able to survive desiccation (reviewed in Ref. [7]; many other reviews cited in Ref. [1]). Although trehalose levels do not always correlate strongly with anhydrobiotic potential in a number of organisms $[1,4,33,34]$, the prevailing hypotheses which attempt to explain anhydrobiosis all involve non-reducing disaccharides in some capacity. Despite this prominence, the current study is the first to examine the genetics of trehalose synthesis in an anhydrobiotic animal. Two tps genes were found in A. avenae, both of which encode very similar proteins and are most similar to tps-2 of the model nematode, C. elegans. No evidence was found for a counterpart in A. avenae of Ce-tps-1, a second gene in C. elegans, but it cannot be ruled out that more genes exist. An unusual feature of the A. avenae tps genes is that four different SL1like spliced leaders are used. Indeed, there might be an even greater degree of variability in spliced leaders of $A$. avenae, since other sequences have been found in a RACE cDNA library (W. Reardon and A.M.B., unpublished). Such variabil- ity is unprecedented in nematode SL1 sequences, which are almost identical across a wide range of species [24], and its significance is currently unclear.

Apart from differences due to alternative splicing, the predicted protein sequences of TPS enzymes from A. avenae are almost identical. Their membership of the GT-20 family of carbohydrate active enzymes is apparent from the conserved sequence, corresponding to $E$. coli OtsA, which comprises the central region of the longer forms of A. avenae TPS sequence. The role of the sequences flanking the GT-20 region is not known, however. Phylogenetic analysis of GT-20 region sequences from a range of organisms suggests that nematode TPS sequences have undergone accelerated molecular evolution. If there are fewer functional constraints on the $\mathrm{N}$ - and C-terminal domains than on the GT-20 region, accelerated evolution might explain the apparently lack of similarity between nematode and other TPS proteins outside the catalytic domain. An understanding of TPS function is complicated by proteins such as yeast ( $S$. cerevisiae) TPS3 and TSL1 which are related to, and form a complex with, the enzymes TPS1 (equivalent to OtsA) and TPS2 (equivalent to OtsB) from the same organism. However, it is thought that TPS 3 and TSL1 have a regulatory, rather than catalytic, role [35]. The N- and C-terminal domains of animal and plant TPS proteins could be involved in protein complex formation and might also have a regulatory function [36], but require further experimental investigation before this can be elucidated.

The increased $A$. avenae tps mRNA levels observed in dehydrating animals is in keeping with a role for trehalose in anhydrobiosis; they are of the same order as, and therefore could be largely responsible for, the concomitant increase in trehalose concentration [3]. Although to a lesser extent, both genes are also upregulated by cold shock and osmotic stress, which might indicate a more general role for trehalose in alleviating the effects of reduced water activity, and perhaps other stresses, in the nematode. Certainly, our data do not support 
an exclusive role for trehalose in anhydrobiosis and, indeed, further evidence, such as might be obtained after RNAi knockdown, is required to demonstrate that it is actually necessary for desiccation tolerance. An increasingly wide range of functions are attributed to trehalose; for example, it is well known to act as an organic osmolyte in micro-organisms [17,37-39] and it has also been implicated in the cold stress response of E. coli [15]. Heat shock and oxidative stress do not seem to activate tps genes in A. avenae, but this contrasts with the situation in micro-organisms, where trehalose is thought to play a role $[13,14,16]$. At present, other than a few studies in anhydrobiosis, there is sparse information on trehalose synthesis in nematodes [40]. In C. elegans, RNAi indicates that trehalose is not required for normal metabolism or development $[9,41]$, but a role in stress tolerance in nematodes seems likely; our tps gene expression results suggest that it will be of interest to determine trehalose concentrations in A. avenae and other nematode species under various stress conditions (other than desiccation [3]).

\section{Acknowledgements}

We would like to thank Drs David Fitzpatrick and Grace McCormack of NUI Maynooth for help with the phylogenetic analysis, and Dr Mark Blaxter (University of Edinburgh) for helpful discussions. This work was supported by grants from the Leverhulme Trust (F/09 717/A), the Isaac Newton Trust, the Royal Irish Academy, the Royal Society and the Charles Slater Fund. A.M.B. is a Science Foundation Ireland Investigator and A.T. is the Anglian Water Fellow in Biotechnology of Pembroke College, Cambridge.

\section{References}

[1] A. Tunnacliffe, J. Lapinski, Resurrecting van Leeuwenhoek's rotifers: a reappraisal of the role of disaccharides in anhydrobiosis, Philos. Trans. R. Soc. Lond. B Biol. Sci. 358 (2003) 1755-1771.

[2] K.A.C. Madin, J.H. Crowe, Anhydrobiosis in nematodes: carbohydrate and lipid metabolism during dehydration, J. Exp. Zool. 193 (1975) 335-342.

[3] J.A. Browne, K.M. Dolan, T. Tyson, K. Goyal, A. Tunnacliffe, A.M. Burnell, Dehydration-specific induction of hydrophilic protein genes in the anhydrobiotic nematode Aphelenchus avenae, Eukaryot. Cell 3 (2004) 966-975.

[4] L.M. Higa, C.Z. Womersley, New insights into the anhydrobiotic phenomenon-the effects of trehalose content and differential rates of evaporative water-loss on the survival of Aphelenchus avenae, J. Exp. Zool. 267 (1993) 120-129.

[5] J. Lapinski, A. Tunnacliffe, Anhydrobiosis without trehalose in bdelloid rotifers, FEBS Lett. 553 (2003) 387-390.

[6] J.M. Aguilera, M. Karel, Preservation of biological materials under desiccation, Crit. Rev. Food Sci. Nut. 37 (1997) 287-309.

[7] A.D. Elbein, Y.T. Pan, I. Pastuszak, D. Carroll, New insights on trehalose: a multifunctional molecule, Glycobiology 13 (2003) 17R27R.

[8] R. Zentella, J.O. Mascorro-Gallardo, P. Van Dijk, J. Folch-Mallol, B. Bonini, C. Van Vaeck, et al., A Selaginella lepidophylla trehalose6-phosphate synthase complements growth and stress-tolerance defects in a yeast tps 1 mutant, Plant Physiol. 119 (1999) 1473-1482.
[9] F.I. Pellerone, S.K. Archer, C.A. Behm, W.N. Grant, M.J. Lacey, A.C. Somerville, Trehalose metabolism genes in Caenorhabditis elegans and filarial nematodes, Int. J. Parasitol. 33 (2003) 1195-1206.

[10] W. Bell, P. Klaassen, M. Ohnacker, T. Boller, M. Herweijer, P. Schoppink, et al., Characterization of the 56-kDa subunit of yeast trehalose6-phosphate synthase and cloning of its gene reveal its identity with the product of CIF1, a regulator of carbon catabolite inactivation, Eur. J. Biochem. 209 (1992) 951-959.

[11] O. Vuorio, N. Kalkkinen, J. Londesborough, Cloning of two related genes encoding the $56 \mathrm{kDa}$ and $123 \mathrm{kDa}$ subunits of trehalose synthase from the yeast Saccharomyces cerevisiae, Eur. J. Biochem. 216 (1993) 849-861.

[12] I. Kaasen, P. Falkenberg, O.B. Styrvold, A.R. Strøm, Molecular cloning and physical mapping of the otsBA genes, which encode the osmoregulatory trehalose pathway of Escherichia coli: evidence that transcription is activated by KatF (AppR), J. Bacteriol. 174 (1994) 889-898.

[13] T. Hottiger, T. Boller, A. Wiemken, Rapid changes of heat and desiccation tolerance correlated with changes in trehalose content in Saccharomyces cerevisiae cells subjected to temperature shifts, FEBS Lett. 220 (1987) 113-115.

[14] M.A. Singer, S. Lindquist, Multiple effects of trehalose on protein folding in vivo and in vitro, Mol. Cell 1 (1998) 639-648.

[15] O. Kandror, A. DeLeon, A.L. Goldberg, Trehalose synthesis is induced upon exposure of Escherichia coli to cold and is essential for viability at low temperatures, Proc. Natl. Acad. Sci. USA 99 (2002) 9727-9732.

[16] N. Benaroudj, D.H. Lee, A.L. Goldberg, Trehalose accumulation during cellular stress protects cells and cellular proteins from damage by oxygen radicals, J. Biol. Chem. 276 (2001) 24261-24267.

[17] A.R. Strøm, Osmoregulation in the model organism Escherichia coli: genes governing the synthesis of glycine betaine and trehalose and their use in metabolic engineering of stress tolerance, J. Biosci. 23 (1998) 437-445.

[18] I.A. Hope (Ed.), C. elegans. A Practical Approach, Oxford University Press, Oxford, UK, 1999.

[19] J.D. Thompson, T.J. Gibson, F. Plewniak, F. Jeanmougin, D.G. Higgins, The CLUSTAL-X windows interface: flexible strategies for multiple sequence alignment aided by quality analysis tools, Nucleic Acids Res. 25 (1997) 4876-4882.

[20] D.L. Swofford, PAUP: Phylogenetic Analysis using Parsimony and Other Methods, Sinauer Associates, Sunderland, MA, 2000. Available from http://paup.csit.fsu.edu.

[21] S. Whelan, N. Goldman, A general empirical model of protein evolution derived from multiple protein families using a maximumlikelihood approach, Mol. Biol. Evol. 18 (2001) 691-699.

[22] H.A. Schmidt, A. von Haeseler, Maximum-likelihood analysis using TREE-PUZZLE, in: A.D. Baxevanis, D.B. Davison, R.D.M. Page, G. Stormo, L. Stein (Eds.), Current Protocols in Bioinformatics, Unit 6.6, Wiley and Sons, New York, 2003.

[23] J.P. Huelsenbeck, F. Ronquist, MRBAYES: Bayesian inference of phylogenetic trees, Bioinformatics 17 (2001) 754-755.

[24] M. Blaxter, L. Liu, Nematode spliced leaders-ubiquity, evolution and utility, Int. J. Parasitol. 26 (1996) 1025-1033.

[25] C. Ray, A.G. Abbott, R.S. Hussey, Trans-splicing of a Meloidogyne incognita mRNA encoding a putative oesophageal gland protein, Mol. Biochem. Parasitol. 68 (1995) 93-101.

[26] H. Koltai, Y. Spiegel, M.L. Blaxter, Regulated use of an alternative spliced leader exon in the plant parasitic nematode Meloidogyne javanica, Mol. Biochem. Parasitol. 86 (1997) 107-110.

[27] R.P. Gibson, J.P. Turkenburg, S.J. Charnock, R. Lloyd, G.J. Davies, Insights into trehalose synthesis provided by the structure of the retaining glucosyltransferase OtsA, Chem. Biol. 9 (2002) 1337-1346.

[28] S. Wong, M.A. Fares, W. Zimmermann, G. Butler, K.H. Wolfe, Evidence from comparative genomics for a complete sexual cycle in the "asexual" pathogenic yeast Candida glabrata, Genome Biol. 4 (2003) R10. 
[29] Y.J. Liu, B.D. Hall, Body plan evolution of ascomycetes, as inferred from an RNA polymerase II phylogeny, Proc. Natl. Acad. Sci. USA 101 (2004) 4507-4512.

[30] M. Blaxter, Caenorhabditis elegans is a nematode, Science 282 (1998) 2041-2046.

[31] G. Vogel, O. Fiehn, L. Jean-Richard-dit-Bressel, T. Boller, A. Wiemken, R.A. Aeschbacher, A. Wingler, Trehalose metabolism in Arabidopsis: occurrence of trehalose and molecular cloning and characterization of trehalose-6-phosphate synthase homologues, J. Exp. Biol. 52 (2001) 1817-1826.

[32] Q. Chen, K.L. Behar, T. Xu, C. Fan, G.G. Haddad, Expression of Drosophila trehalose-phosphate synthase in HEK-293 cells increases hypoxia tolerance, J. Biol. Chem. 278 (2003) 49113-49118.

[33] N.B. Hendriksen, Anhydrobiosis in nematodes: studies on Plectus sp, in: P. Lebrun, H.M. André, A. De Medts, C. Grégoire-Wibo, G. Wauthy (Eds.), Proceedings of the Eigth International Colloquium of Soil Zoology, Dieu-Brichart, Ottignies-Louvain-la-Neuve, Belgium, 1983, pp. 387-394.

[34] J. Dijksterhuis, R.A. Samson, Food and crop spoilage on storage, in: F. Kempken (Ed.), The Mycota XI: Agricultural Applications, Springer-Verlag, Berlin, 2002, pp. 39-52.
[35] W. Bell, W. Sun, S. Hohmann, S. Wera, A. Reinders, C. De Virgilio, et al., Composition and functional analysis of the Saccharomyces cerevisiae trehalose synthase complex, J. Biol. Chem. 273 (1998) 33311-33319.

[36] P. Van Dijck, J.O. Mascorro-Gallardo, M. De Bus, K. Royackers, G. Iturriaga, J.M. Thevelein, Truncation of Arabidopsis thaliana and Selaginella lepidophylla trehalose-6-phosphate synthase unlocks high catalytic activity and supports high trehalose levels on expression in yeast, Biochem. J. 366 (2002) 63-71.

[37] P.I. Larsen, L.K. Sydnes, B. Landfald, A.R. Strøm, Osmoregulation in Escherichia coli by accumulation of organic osmolytes: betaines, glutamic acid, and trehalose, Arch. Microbiol. 147 (1987) 1-7.

[38] E.C.A. Eleutherio, F.M. Maia, M.D. Pereira, R. Degre, D. Cameron, A.D. Panek, Induction of desiccation tolerance by osmotic treatment in Saccharomyces uvarum var. carlsbergensis, Can. J. Microbiol. 43 (1997) 495-498.

[39] C.-G. Hounsa, E.V. Brandt, J. Thevelein, S. Hohmann, B.A. Prior, Role of trehalose in survival of Saccharomyces cerevisiae under osmotic stress, Microbiology 144 (1998) 671-680.

[40] C.A. Behm, The role of trehalose in the physiology of nematodes, Int. J. Parasitol. 27 (1997) 215-229.

[41] R.S. Kamath, A.G. Fraser, Y. Dong, G. Poulin, R. Durbin, M. Gotta, et al., Systematic functional analysis of the Caenorhabditis elegans genome using RNAi, Nature 421 (2003) 231-237. 